\title{
An unusual etiology of torsade de pointes-induced syncope
}

Marco Morosin ${ }^{1}$, Ermanno Dametto², Federica Del Bianco ${ }^{2}$, Marco Brieda², Gian L. Nicolosi² $^{2}$

${ }^{1}$ Cardiovascular Department "Ospedali Riuniti” Trieste and Postgraduate School Cardiovascular Sciences, University of Trieste and Cardiology Department "Santa Maria degli Angeli" Hospital, Pordenone, Italy

${ }^{2}$ Cardiology Department, ARC. "Santa Maria degli Angeli" Hospital, Pordenone, Italy

Submitted: 23 March 2015

Accepted: 23 May 2015

Arch Med Sci 2017; 13, 3: 686-688

DOI: https://doi.org/10.5114/aoms.2017.67287

Copyright $\odot 2017$ Termedia \& Banach

An 80-year-old woman was admitted to our Emergency Department because of syncope. The patient had no family history of sudden deaths or a medical history of depressive disorder, and she did not smoke or use illicit drugs. In the previous year, her daily medications included acetylsalicylic acid, delapril, simvastatin, vitamin $D_{3}$ and a daily dose of $50 \mathrm{mg}$ of fluvoxamine. The patient was lucid and cooperative and reported taking all tablets regularly. Repeated episodes of dizziness and transient loss of consciousness (T-LOC) occurred in the months preceding admission. At 4 p.m. on the day of admission, the patient had another episode of T-LOC while sitting on the bed, witnessed by her husband. At the Emergency Department, telemetry recorded in lead III several runs of self-terminating torsade de pointes (TdP) ventricular tachycardia with concomitant T-LOC (Figure 1). Prompt administration of IV magnesium ( $1 \mathrm{~g}$ IV bolus followed by continuous infusion of $0.5 \mathrm{~g} / \mathrm{h}$ ) and IV lidocaine (50 mg IV bolus followed by maintenance IV infusion of $1 \mathrm{mg} / \mathrm{min}$ ) was performed. Physical examination was unremarkable. A 12-lead electrocardiogram (ECG) showed sinus rhythm, 59/min, incomplete left bundle branch block and a prolonged rate-corrected QT interval (QTc) of $0.51 \mathrm{~s}$ (Figure 2). We measured the QT interval from the onset of the QRS complex to the end of the T wave and used Bazett's formula to correct the QT interval: QTC $=\mathrm{QT} /(\mathrm{R}-\mathrm{R}$ interval) $1 / 2$ [1]. Blood tests, electrolytes included, were normal. Transthoracic echocardiogram showed a left ventricle normal in size, moderate ventricular hypertrophy and normal regional function. Left ventricular ejection fraction was $54 \%$. Color Doppler examination revealed mild mitral regurgitation and mild to moderate tricuspid regurgitation. Coronary angiography ruled out significant coronary artery disease (stenosis > 50\%). Although the patient was not taking any known QT-prolonging medications, fluvoxamine was believed to be the likely cause of the prolonged QT interval and the malignant arrhythmia. Fluvoxamine was thus discontinued and in the subsequent $36 \mathrm{~h}$ the patient had three other TdP episodes, one of them requiring a DC shock. In the next days, serial ECGs indicated a stable sinus rhythm with progressive normalization of the QTC interval. No other ventricular arrhythmias were observed. The patient was discharged home and instructed not to take fluvoxamine. After a month, a 7-day ambulatory ECG recorded a single episode of an 18-beat run of self-terminating ventricular tachycardia at $130 \mathrm{bpm}$. The decision was made to place an implantable cardioverter-defibrillator (ICD). At 3 months' follow-up, the

\author{
Corresponding author: \\ Marco Morosin MD \\ Cardiovascular Deptartment \\ Ospedali Riuniti Trieste \\ Marco Morosin \\ Via Valdoni 7 \\ 34149 Trieste, Italy \\ Phone: +390403994477 \\ E-mail: marco.morosin01@ \\ gmail.com
}




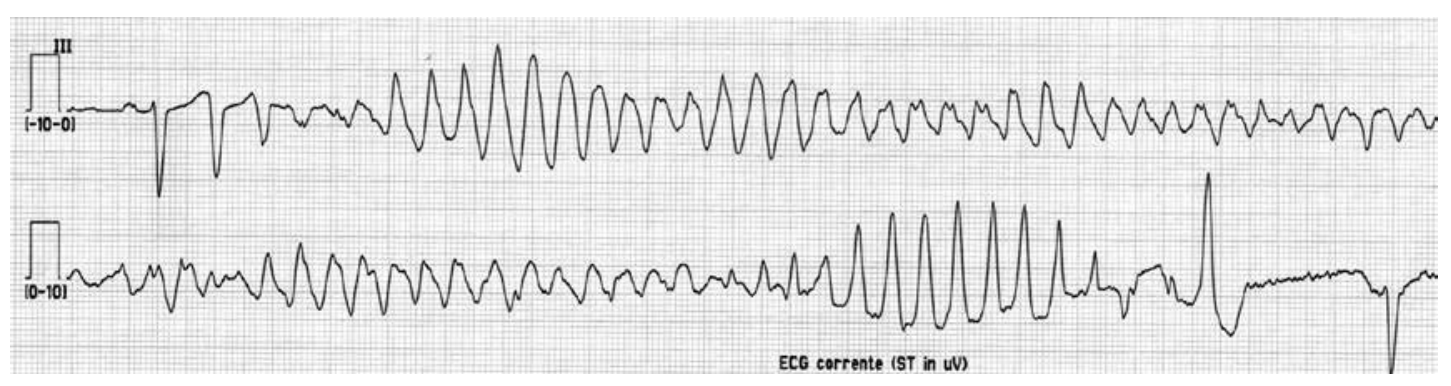

Figure 1. ECG at admission, showing torsade de pointes ventricular tachycardia in lead III

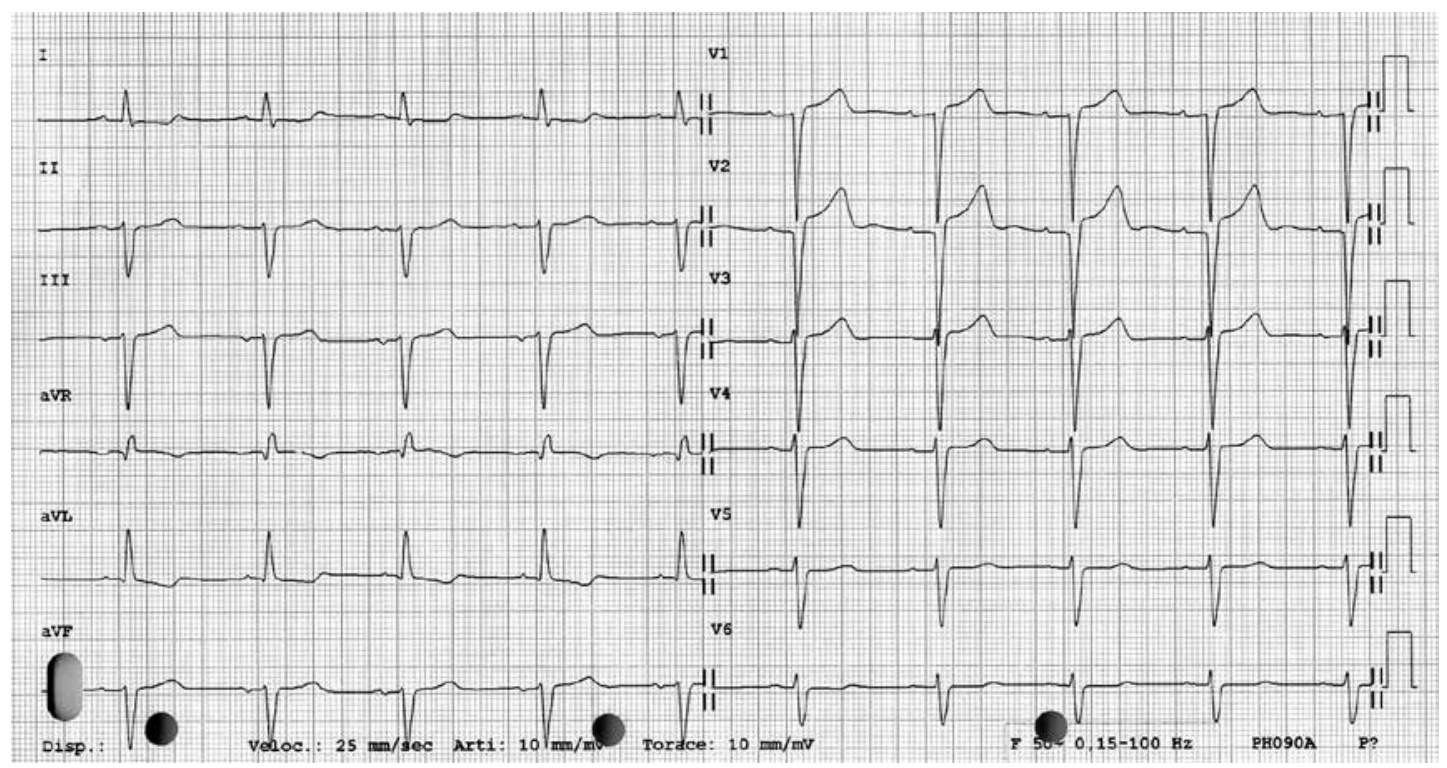

Figure 2. A 12-lead ECG showing the prolonged QTc interval of $0.51 \mathrm{~s}$

patient reported no T-LOC, no dizziness and no palpitations. On ICD remote monitoring no further TdP were reported.

To our knowledge, this is the first strong report of TdP probably [2] induced by fluvoxamine at therapeutic doses. In contrast to previous studies $[3,4]$ in which fluvoxamine did not seem to be a QTc-prolonging drug, we obtained evidence, consistently with Nia et al. [5], of a patient on fluvoxamine therapy with a prolonged QTc of $0.51 \mathrm{~s}$, which shortened after drug withdrawal.

In general, the presence of a prolonged QTC interval on the ECG in a patient who is admitted to the hospital because of syncope represents an independent predictor of long-term mortality [6]. The majority of QT prolonging drugs block the cardiac rapidly activating delayed rectifier potassium current $\left(I_{k r}\right)$, whose major subunit is encoded by the human ether-a-go-go related gene (HERG) $[7,8]$. Milnes et al. [9] reported that fluvoxamine inhibits HERG potassium channels with rapid onset and its inhibition is only partially reduced in F656A mutant channels, which are known to profoundly attenuate the inhibition of HERG channels by many known QT prolonging drugs. This suggests that the pharmacological mechanism of fluvoxamine's effect on HERG channels is different from that of other QT prolonging agents [9]. Individual genetic susceptibility might have played a role in the development of such drug-induced QT prolongation. Indeed, polymorphisms have been identified in HERG, and some of them are now known to increase the risk of TdP [10]. Of note, female patients with long QT syndrome show significantly higher $\mathrm{KCNH} 2 \mathrm{mRNA}$ levels compared with male patients, indicating a higher female risk of cardiovascular events [11].

Finally, one would argue the potential advantages of using a wearable cardioverter-defibrillator (WCD) in this patient [12]. Nevertheless, we were quite confident that once fluvoxamine was stopped and QTc normalized on ECG before discharge, the patient would not experience other major ventricular arrhythmias in the short term. Moreover, there are not enough data on the use of a WCD in this context, and in fact WCD still represents a possible future indication for drug-induced QT prolongation [13].

This case report therefore has the aim to better focus and sensitize physicians to this potential- 
ly life-threatening, albeit very rare, possible side effect. Patients on fluvoxamine treatment should be monitored closely for QT/QTc interval prolongation with serial ECG. Considering the half life of fluvoxamine, a reasonable timing for serial ECG might be: basal ECG before fluvoxamine administration, and after drug administration at 2-3 days, 7-8 days and again at 10-15 days, once steadystate plasma levels of fluvoxamine are achieved.

We have to acknowledge a potential limitation to this report. As we cannot determine plasma concentration of fluvoxamine at admission, we fully relied on the patient's statement of taking all medications regularly.

In conclusion, regarding our report and evidence indicating fluvoxamine-mediated potassium channel inhibition, further investigations about the potential risk of fluvoxamine-induced arrhythmia are warranted.

\section{Conflict of interest}

The authors declare no conflict of interest.

\section{References}

1. Bazett HC. An analysis of the time-relations of electrocardiograms. Heart 1920; 7: 353-70.

2. Edwards IR, Aronson JK. Adverse drug reactions: definitions, diagnosis, and management. Lancet 2000; 356 1255-9.

3. Wenzel-Seifert K, Wittmann M, Haen E. QTc prolongation by psychotropic drugs and the risk of torsade de pointes. Dtsch Arztebl Int 2011; 108: 687-93.

4. van Noord C, Straus SM, Sturkenboom MC, et al. Psychotropic drugs associated with corrected QT interval prolongation. J Clin Psychopharmacol 2009; 29: 9-15.

5. Nia AM, Dahlem KM, Gassanov N, Hungerbühler $H$, Fuhr U, Er F. Clinical impact of fluvoxamine-mediated long QTU syndrome. Eur J Clin Pharmacol 2012; 68: 109-11.

6. Balasubramaniyam N, Palaniswamy C, Aronow WS, et al. Association of corrected QT interval with longterm mortality in patients with syncope. Arch Med Sci 2013; 9: 1049-54.

7. Sanguinetti MC, Jlang C, Curran ME, Keating MT. A mechanistic link between an inherited and an acquired cardiac arrhythmia: HERG encodes the IKr potassium channel. Cell 1995; 81: 299-307.

8. Trudeau MC, Warmke JW, Ganetzky B, Robertson GA. HERG, a human inward rectifier in the voltage-gated potassium channel family. Science 1995; 269: 92-5.

9. Milnes JT, Crociani O, Arcangeli A, Hancox JC, Witchel HJ. Blockade of HERG potassium currents by fluvoxamine: incomplete attenuation by $\mathrm{S} 6$ mutations at $\mathrm{F} 656$ or Y652. Br J Pharmacol 2003; 139: 887-98.

10. Fitzgerald PT, Ackerman MJ. Drug-induced torsades de pointes: the evolving role of pharmacogenetics. Heart Rhythm 2005; 2 (2 Suppl): S30-7.

11. Moric-Janiszewska E, Głogowska-Ligus J, Paul-Samojedny M, Węglarz L, Markiewicz-Łoskot G, Szydłowski L. Age- and sex-dependent mRNA expression of KCNQ1 and HERG in patients with long QT syndrome type 1 and 2. Arch Med Sci 2011; 7: 941-7.
12. Meyer C, Carvalho P, Brinkmeyer C, Kelm M, Couceiro R, Mühlsteff J. Wearable sensors in syncope management. Med Sci Monit 2015; 21: 276-82.

13. Klein HU, Goldenberg I, Moss AJ. Risk stratification for implantable cardioverter defibrillator therapy: the role of the wearable cardioverter-defibrillator. Eur Heart J 2013; 34: 2230-42. 